\title{
Recombinant adeno-associated virus serotype 9 in a mouse model of atherosclerosis: Determination of the optimal expression time in vivo
}

\author{
QINGJIE CHEN $^{1,2^{*}}$, HUI ZHAI ${ }^{1,2^{*}}$, XIAOMEI LI ${ }^{1,2}$, YITONG MA ${ }^{1,2}$, BANGDANG CHEN $^{2,3}$, FEN LIU $^{2,3}$, \\ HONGMEI LAI ${ }^{1,2,4}$, JIA XIE ${ }^{1,2,5}$, CHUNHUI HE ${ }^{1,2}$, JUNYI LUO ${ }^{1,2}$, JING GAO ${ }^{1,2}$ and YINING YANG Y $^{1,2}$ \\ ${ }^{1}$ Department of Cardiology, First Affiliated Hospital of Xinjiang Medical University; \\ ${ }^{2}$ Xinjiang Key Laboratory of Cardiovascular Disease Research; ${ }^{3}$ Clinical Research Institute of Xinjiang Medical University, \\ Urumqi, Xinjiang 830054; ${ }^{4}$ Department of Cardiology, People's Hospital of Xinjiang Uygur Autonomous Region, Urumqi, \\ Xinjiang 830001; ${ }^{5}$ Department of Cardiology, Second People's Hospital of Nanyang, Nanyang, Henan 473012, P.R. China
}

Received November 23, 2015; Accepted November 23, 2016

DOI: $10.3892 / \mathrm{mmr} .2017 .6235$

\begin{abstract}
Adeno-associated virus 9 (AAV9) has been identified as one of the optimal gene transduction carriers for gene therapy. The aim of the present study was to determine the gene transfection efficiency and safety of an AAV9 vector produced using a recombinant baculovirus ( $\mathrm{rBac}$ )-based system. AAV9-cytomegalovirus (CMV)-green fluorescent protein was produced using an rBac system and the resulting vector particles were injected intravenously into mice. Animals were sacrificed at 14, 21, 28, 35, 60, 90 and 120 days following injection. GFP expression in aortic vasculature and aortic plaques in $\mathrm{C} 57 / 6 \mathrm{~B}$ and apolipoprotein $\mathrm{E}^{-/-}$mice was analyzed by fluorescence imaging and western blotting. In vivo analyses of biological markers of liver and heart damage, and renal function, as well as in vitro terminal deoxynucleotidyl transferase dUTP nick end labeling analysis were used to determine the toxicity of the AAV9 carrier. The findings of the present study demonstrated that AAV9 viral vectors packaged using the rBac system functioned appropriately in arteriosclerosis plaques. The CMV promoter significantly induced GFP expression in the vascular plaque in a time-dependent manner. AAV9-CMV viral particles did not lead to heart, liver or renal damage and no change in apoptotic rate was identified. These
\end{abstract}

Correspondence to: Professor Yining Yang or Professor Yitong Ma, Department of Cardiology, The First Affiliated Hospital of Xinjiang Medical University, 137 Liyushan South Road, Urumqi, Xinjiang 830054, P.R. China

E-mail: yangyn5126@163.com

E-mail: myt-xj@163.com

*Contributed equally

Key words: AAV9, vascular, atherosclerotic plaques, GFP expression, rBac-based system findings indicated that AAV9-CMV may be effectively and safely used to transfect genes into atherosclerotic plaques.

\section{Introduction}

Cardiovascular disease is the leading cause of mortality in Western countries and $\sim 75 \%$ of these cases may be attributed to atherosclerosis (1). Unstable atherosclerotic plaque rupture may form occlusive thrombi that block coronary arteries, which may lead to myocardial infarction. Currently, no effective therapeutic agents have been developed to stabilize vulnerable plaques; thus, it is necessary to develop novel methods and tools to investigate and treat vulnerable plaques. Gene therapy may be a promising novel strategy for addressing this clinical problem. Local gene therapy using adenoviral vectors in the vascular lumen in animal models has been identified to improve the composition of plaques; however, surgery is required to administer the treatment (2-4). Gene intervention aimed at preventing unstable atherosclerotic plaque rupture appears to be highly effective when a vector that specifically targets gene expression in the plaque is injected intravenously. This approach requires determining the appropriate therapeutic dose to provide localized treatment of the correct targets. Previous studies have demonstrated that in the adeno-associated virus 2 (AAV2) platform, the peptides are exposed on the viral capsid surface, enabling effective receptor binding at a plaque; therefore, this is a potentially effective targeting vector $(5,6)$. Atherosclerosis is a chronic disease, thus, there is a potential need for long-term gene expression. However, to the best of our knowledge, no previous study has reported on vectors that specifically target atherosclerotic plaques at different time points to identify the optimal conditions for transfection efficiency and safety.

AAV2 is used in various gene therapies due to its various desirable properties, including in vivo induction of gene expression for $>10$ months (7). However, following systemic administration of AAV2, the vascular cell transfection efficiency was low $(8,9)$ and a large quantity of the virus was located in the liver and spleen $(10,11)$. When AAV2 was 
compared with other serotypes $(12,13)$, it was observed that following intravascular injection, the AAV1, AAV6, AAV8 and AAV9 serotypes passed more efficiently through the endothelial barrier and were more effective at targeting organ gene expression (14-18). Among these novel AAV serotypes, AAV serotype 9 (AAV9) has been identified as an attractive vector based on its superior performance in transduction of the muscles, heart and lungs $(16,17,19)$. Due to its wide range of tissue tropism, particularly in the liver, when an AAV vector is transfected via the intravascular route, the liver may act as a large storage location. Tissue-specific promoters have also been used to reduce unintended gene intervention; however, their efficiency is lower compared with that of non-specific promoters, such as the cytomegalovirus (CMV) promoter (20). Previous studies have focused on identifying effective AAV serotypes for cardiac or hepatic gene therapy $(16,18,20,21)$; however, to the best of our knowledge, there has been no investigation on AAV9 gene transfer driven by CMV in apolipoprotein $\mathrm{E}^{-/-}\left(\mathrm{ApoE}^{-/}\right)$mice that are prone to form plaques at different time points.

The most common method for producing recombinant AAV9 (rAAV9) is by using packaging plasmids in HEK293 cells, however, the traditional production process is limited by the difficulty in producing a sufficient number of vectors for large-scale pre-clinical testing and clinical trials, thereby hindering the progression of gene therapy (22). Recombinant baculovirus (rBac)-based systems may produce a large number of AAV vectors (22-24), thus increasing potential of clinical gene therapy. However, the biological information regarding rAAV9 vectors produced using an rBac system remains to be fully elucidated. Thus, the present study aimed to evaluate whether rAAV9 with the CMV promoter produced using the rBac system may be systemically transduced into $\mathrm{ApoE}^{-/-}$mice, that are vulnerable to plaque formation and to determine the transfection efficiency, safety profile and a timeline of transduced gene expression.

\section{Materials and methods}

Vector design. The AAV9 recombinant vector was purchased from Virovek (Hayward, CA, USA) and produced using the rBac-based system in SF9 cells, as previously described (23-25). rAAV9 vectors were composed of single-stranded DNA containing the enhanced green fluorescent protein (GFP) gene and driven by the human cytomegalovirus (CMV) promoter (rAAV9-CMV-GFP). Vector titers were determined using a quantitative polymerase chain reaction (qPCR) according to a previously described method $(17,26)$ with primers corresponding to the CMV enhancer region.

Animals. A total of $40 \mathrm{C} 57 \mathrm{BL} / 6$ and $40 \mathrm{ApoE}^{-/-}$male mice (weight, 18-22 g) were bred and housed in a specific pathogen-free barrier facility at $22-25^{\circ} \mathrm{C}$ and were purchased from the Peking University Health Science Center (Beijing, China). Approval for animal studies was obtained from the Ethics Committee of the First Affiliated Hospital of Xinjiang Medical University (Urumqi, China). All the mice used in the present study were aged 8 weeks. The mice were housed in the Xinjiang Medical University Animal Center with a 12-h light/dark cycle with free access to food and water. C57BL/6 mice were maintained on a normal diet for 16 weeks, and $\mathrm{ApoE}^{-/-}$mice received a high-fat diet $(0.25 \%$ cholesterol and $15 \%$ cocoa butter) for 16 weeks. After 16 weeks, $35 \mathrm{C} 57 \mathrm{BL} / 6$ and $35 \mathrm{ApoE}^{-/-}$mice were randomly selected for the gene transfection experiments. Mice were anesthetized with a mixture containing $100 \mathrm{mg} / \mathrm{kg}$ ketamine, $20 \mathrm{mg} / \mathrm{kg}$ xylazine and $1.2 \mathrm{mg} / \mathrm{kg}$ atropine administered by intraperitoneal injection. Subsequently, the animals received a tail vein injection of $100 \mu \mathrm{l}$ saline containing 5.0x10 11 rAAV9-CMV-GFP vector particles. The transduction dose was similar to that in previous studies, which determined that this dose induced a specific and efficient transgene expression in the aorta and in atherosclerotic plaques $(27,28)$. The remaining 5 C57BL/6 and $5 \mathrm{ApoE}^{-/-}$mice were injected with $0.09 \%$ saline and served as the control group.

Mice were sacrificed following anesthesia with $100 \mathrm{mg} / \mathrm{kg}$ ketamine, $20 \mathrm{mg} / \mathrm{kg}$ xylazine and $1.2 \mathrm{mg} / \mathrm{kg}$ atropine by intraperitoneal injection at 14, 21, 28, 35, 60, 90 and 120 days following virus administration. A total of 5 mice aorta specimens were acquired at each time point. Prior to sacrifice the mice were fasted for $12 \mathrm{~h}$. Blood samples were collected by cardiac puncture at the time of sacrifice and the serum was separated by centrifugation at $600 \times g$ for $10 \mathrm{~min}$ at $4^{\circ} \mathrm{C}$. The aorta was isolated from the aortic arch to the iliac bifurcation. The aorta, heart, and liver were immediately frozen in liquid nitrogen and stored at $-80^{\circ} \mathrm{C}$ for further molecular analysis or fresh frozen for histological studies. The brachiocephalic artery was also removed, immersed in $4 \%$ formaldehyde overnight at $4^{\circ} \mathrm{C}$, embedded in optimal cutting temperature (OCT) compound (Sakura Finetek USA, Inc., Torrance, CA, USA), and stored at $-80^{\circ} \mathrm{C}$.

Quantification of biological markers. At 14, 21, 28, 35, 60, 90 and 120 days after vector transduction, blood was collected from the mice via cardiac puncture for subsequent biochemical testing. Plasma levels of lactate dehydrogenase (LDH), and creatine kinase (CK) for cardiac toxicity, aspartate aminotransferase (AST) and alanine aminotransferase (ALT) for liver toxicity, along with creatinine and urea nitrogen (BUN) for renal function were determined using an automatic biochemical analyzer (HITACHI-7600; Hitachi, Ltd., Tokyo, Japan).

Laser confocal microscopy. The mice were anesthetized and subsequently blood samples were collected via cardiac puncture from the left ventricle using a heparin-coated syringe. Subsequently, the left common carotid artery and the aortic root were excised, immersed in $4 \%$ formaldehyde overnight at $4^{\circ} \mathrm{C}$, and embedded in OCT compound. The entire length of the common carotid artery underwent histological analysis to ascertain the transfection efficiency of the rAAV9-CMV-GFP in atherosclerotic plaques. To eliminate background autofluorescence, Sky Blue 6B (cat. no. C8679; Sigma-Aldrich; Merck Millipore, Darmstadt, Germany) was used. Frozen sections were viewed by confocal fluorescence microscopy to identify GFP expression. In the present study, OCT compound-embedded carotid artery samples were cross-sectioned into $6-\mu \mathrm{m}$ thick pieces at 50- $\mu \mathrm{m}$ intervals. A total of 5 cross-sections from each mouse were analyzed by confocal fluorescence microscopy. The whole cross-section was viewed in one field for the quantitative measurements and the GFP expression percentage values were averaged (mean \pm standard deviation). 
An automated image analysis system (Image-Pro Plus version 5.0; Media Cybernetics, Inc., Rockville, MD, USA) was used for the quantitative measurements. The GFP-positive area was quantified by computer-assisted color-gated measurements and the ratio of the positive staining area to the plaque area was also calculated.

Western blot analysis. Protein extracts from fresh carotid plaques were separated on $12 \%$ SDS-polyacrylamide gels and transferred to polyvinylidene fluoride membranes. Membranes were blocked with 5\% non-fat milk, then washed with PBS containing $0.1 \%$ Tween 20 and incubated with an appropriate primary antibody at $4^{\circ} \mathrm{C}$ overnight. The blots were probed with antibodies against GAPDH (1:1,000; catalog no. $2118 \mathrm{~S}$; Cell Signaling Technology, Inc., Danvers, MA, USA) and GFP (1:1,000; catalog no. 2956S; Cell Signaling Technology, Inc.). Following an overnight incubation, the blots were washed with Tris-buffered saline-Tween-20 and incubated with an alkaline phosphatase-conjugated secondary antibody at room temperature for $2 \mathrm{~h}$ (catalog no. WP20007; Invitrogen; Thermo Fisher Scientific, Inc., Waltham, MA, USA), and then washed again. The blots were then visualized using diaminobenzidine. Image $\mathrm{Lab}^{\mathrm{TM}}$ software version 4.0 (Bio-Rad Laboratories, Inc., Hercules, CA, USA) was used for densitometry. The results were calculated as GFP gray value/GAPDH gray value.

Apoptosis evaluation. To evaluate whether the rAAV9 vector transduction resulted in increased cytotoxicity, cardiomyocytes and hepatocytes were seeded at a density of $3 \times 10^{5}$ cells/ well in 12-well plates, treated with rAAV9-CMV-GFP, and cultured for 4 days prior to a terminal deoxynucleotidyl transferase dUTP nick end labeling (TUNEL) assay. Images were captured using the Leica DM4000 Inverted fluorescence microscope (Leica Microsystems GmbH, Wetzlar, Germany) and the results were analyzed by Image $\mathbf{J}$ software version 5.0 (National Institutes of Health, Bethesda, MD, USA). Untreated cells were used as the control group.

Statistical analysis. Data are presented as the mean \pm standard deviation. Factorial analysis of variance was conducted to analyze the individual and interactive effects. Multiple comparisons were analyzed by least significant difference post-hoc tests. All the analyses were performed using SPSS version 22.0 (IBM SPSS, Armonk, NY, USA) and $\mathrm{P}<0.05$ was considered to indicate a statistically significant difference.

\section{Results}

Quantitative analysis of experimental model. A total of 40 C57BL/6 and $40 \mathrm{ApoE}^{-/-}$mice were divided into 8 groups. The experiments were performed without eliminating any mice, data from all the mice were included in the analysis. The experimental procedural flow has been presented in Fig. 1.

Time course of GFP expression in the aorta of C57BL/6 mice. To eliminate background autofluorescence, Sky Blue 6B was used on frozen aorta sections from $\mathrm{C} 57 \mathrm{BL} / 6$ and $\mathrm{ApoE}^{-/-}$mice and vasculature and atherosclerotic plaque autofluorescence were successfully eliminated. GFP fluorescence in the carotid plaques was detected at 35 days as presented in Fig. 2.
In C57BL/6 mice, vascular cryosections were viewed by confocal fluorescence microscopy to identify GFP expression at different time points. Vascular background autofluorescence was eliminated by the use of Sky Blue 6B; GFP fluorescence was not detected in the carotid vasculature at 14, 21, 35, 60, 90, or 120 days after transfection.

Western blot analysis of GFP in the aorta of C57BL/6 mice. Aorta vasculature tissues were used for western blot analysis to detect GFP expression at different time points, 5 mice were analyzed at each time point. GFP was detected at different time points after rAAV9-GFP transfection. GFP expression level gradually increased from day 14 onwards, remaining at a persistently low level, and was reduced at day 120 . GFP expression was significantly increased at 21, 28, 35, 60 and 90 days (Fig. 3; $\mathrm{P}<0.05$ vs. 14 days).

Time course of GFP expression in aortic plaques in ApoE-/mice. In $\mathrm{ApoE}^{-/-}$mice, the carotid atherosclerotic plaque cryosections were observed using a confocal fluorescence microscope to identify GFP expression at different time points. GFP fluorescence in carotid vascular plaques was detected at 14, 21, 35, 60, 90 and 120 days after transfection. GFP fluorescence in atherosclerotic plaques steadily increased from 14 days after transfection. The highest strongest GFP fluorescence was detected at 35 days after transfection in the atherosclerotic plaques, with a transfection efficiency of $\sim 15 \%$. Subsequently, the fluorescence intensity decreased, as presented in Fig. 4A and $\mathrm{B}(\mathrm{P}<0.05$ vs. previous time point). GFP expression in aorta vascular tissues was detected by western blotting at different time points and 5 mice were analyzed at each time point. GFP expression was detected at different time points and it increased with time. GFP expression peaked at 35 days after transfection. Subsequently, GFP expression was reduced, as presented in Fig. 4C and D $(\mathrm{P}<0.05$ vs. previous time point).

Safety analysis. In order to determine the safety of this procedure, serum levels of LDH, AST, ALT, CK, creatinine and $\mathrm{BUN}$ were determined at different time points in $\mathrm{ApoE}^{-/-}$mice. No significant difference was identified in the levels of these myocardial enzymes and markers of liver and kidney function in treated $\mathrm{ApoE}^{-/-}$mice compared with control mice, as presented in Fig. 5. At 4 days after viral transfection, cell apoptotic rate was analyzed using TUNEL assay. No significant difference was identified in the number of TUNEL-positive cardiomyocytes and hepatic cells compared with non-transfected control cells, as presented in Fig. 6.

\section{Discussion}

The primary challenge of using AAV vectors in clinical trials is the inability to produce a sufficient quantity of high purity vectors. The rBac-SF9 system has a 10-fold higher cost performance compared with the HEK293 system in producing AAV vectors (24). rAAV9 vectors produced using the rBac system have been widely used, it is critical to determine whether vectors produced using this platform have efficient infectivity, appropriate distribution, long-term expression and an adequate safety profile. The present study 


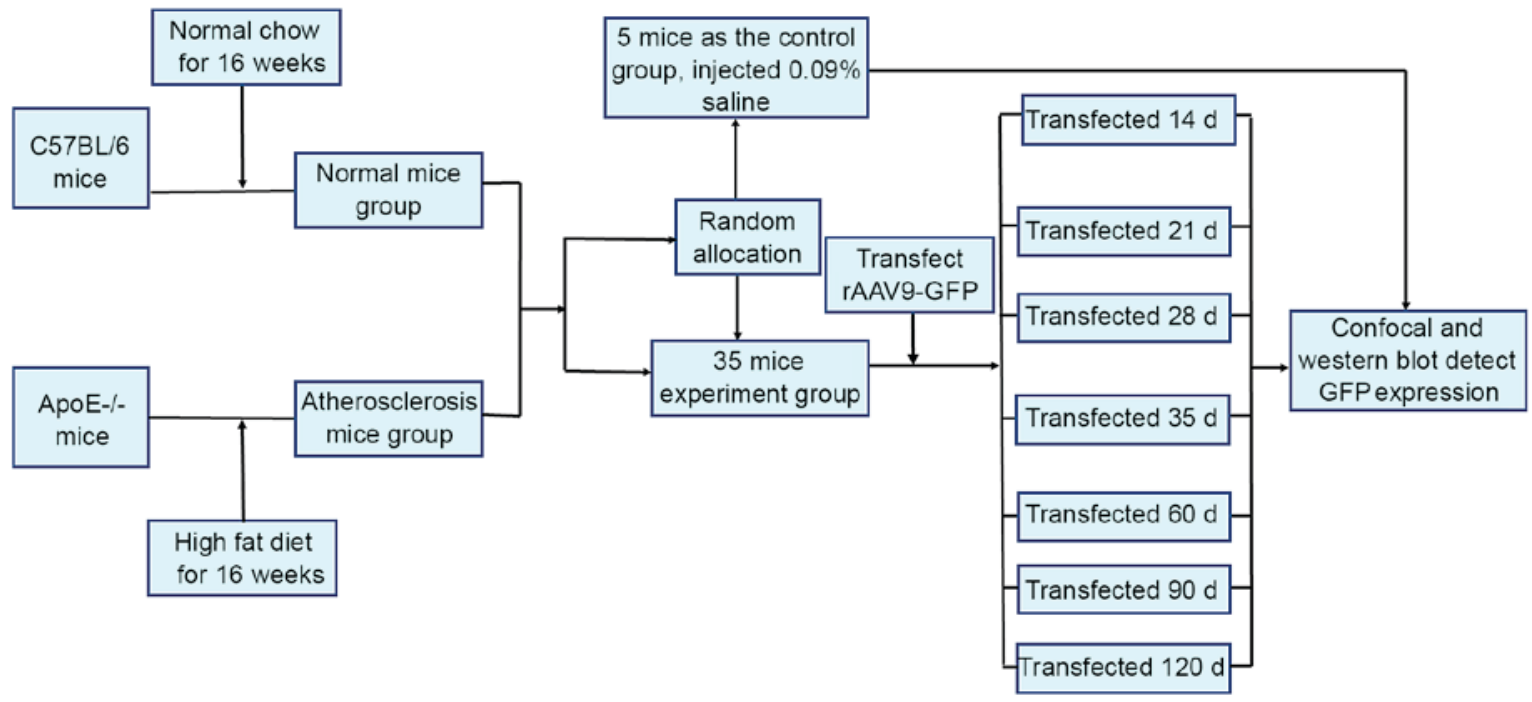

Figure 1. Flow chart of experimental design. ApoE, apolipoprotein E; rAAV9, recombinant adeno-associated virus 9; GFP, enhanced green fluorescent protein.
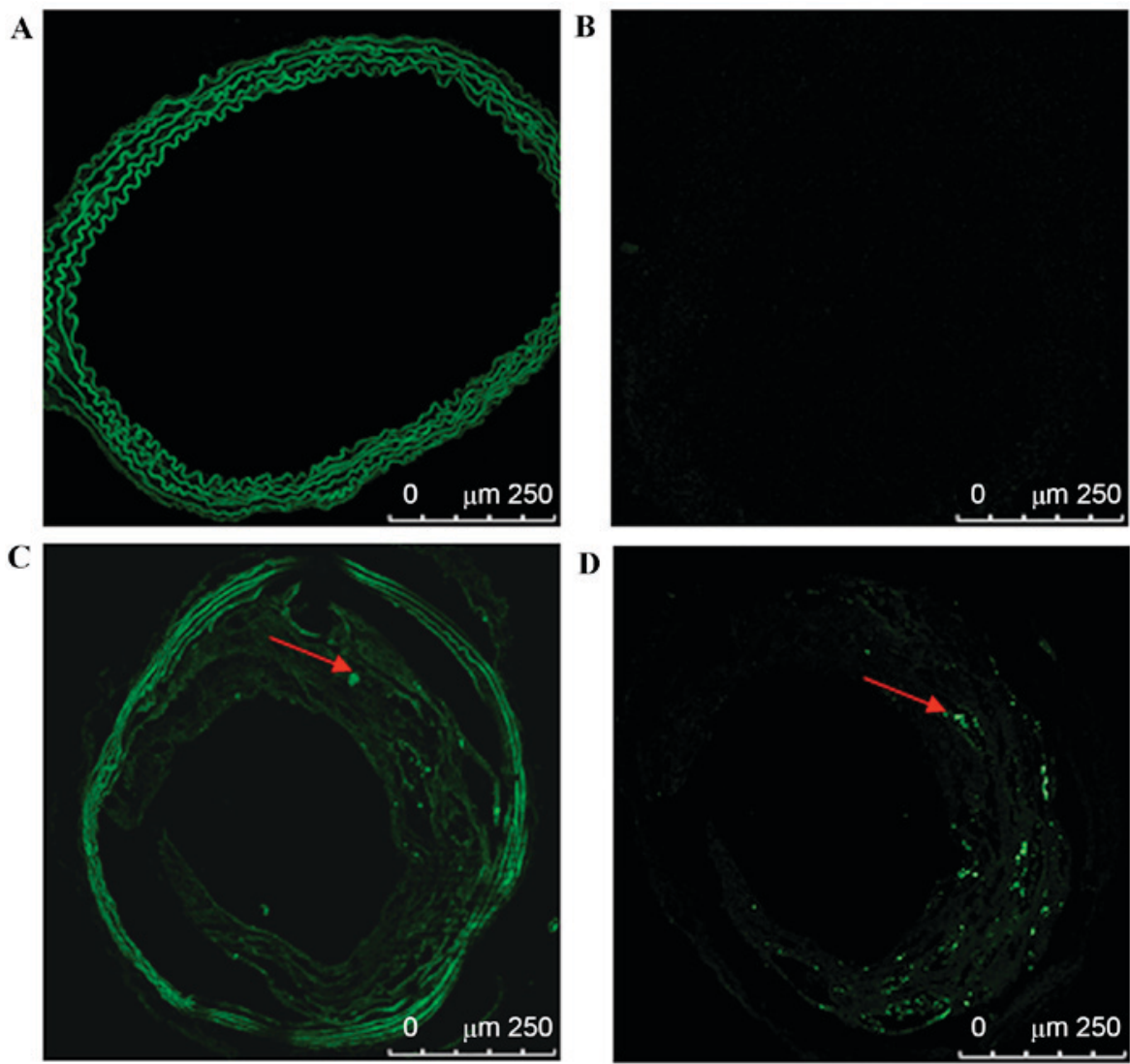

Figure 2. AAV9-mediated GFP expression in vivo. Representative confocal microscopy images of AAV9-CMV vector-mediated GFP expression in the carotid artery and in carotid atherosclerotic plaques after 35 days. GFP expression was detected in C57/6B control mice (A) without and (B) with Sky Blue 6B. GFP expression in carotid atherosclerotic plaques in $\mathrm{ApoE}^{-/}$mice was detected (C) without and (D) with Sky Blue 6B. Scale bar, $250 \mu \mathrm{m}$. The red arrows indicate areas of GFP expression, which are green. AAV9, adeno-associated virus 9; GFP, green fluorescent protein; CMV, cytomegalovirus; ApoE, apolipoprotein E.

determined that the rAAV9 viral vectors produced using the rBac system were effective in vivo and induced enhanced GFP transgene expression predominantly in $\mathrm{ApoE}^{-/-}$mouse carotid atherosclerotic plaques for up to 120 days. In addition, the transfected rAAV9 vectors did not lead to major organ damage, including cardiomyocyte and hepatic cell injury, and apoptosis.
Sky blue solution was used to eliminate vascular tissue autofluorescence, which may be detected when localizing the GFP reporter gene and protein expression by immunofluorescence. Green immunofluorescence in the C57BL/6 aorta vasculature was low when the animals were infected with rAAV9-CMV-GFP at a high dose of 5.0x10 11 virus genomes/mouse at different time periods. Additionally, GFP 

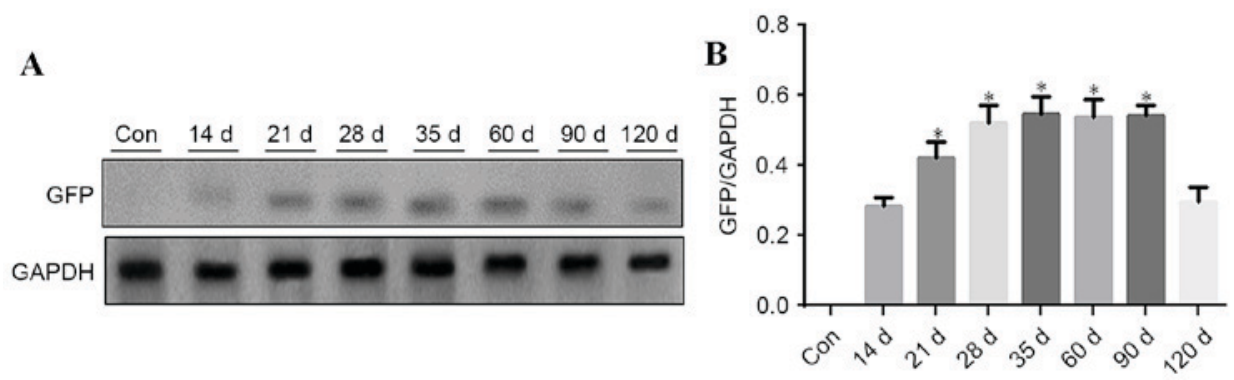

Figure 3. Temporal changes in AAV9-mediated GFP expression in the carotid artery in C57/6B mice. (A) GFP expression was quantified using western blotting at different time points in the carotid artery following a viral injection ( $\mathrm{n}=5$ per group). (B) Quantitative analysis of GFP protein expression levels. The expression of the GFP protein was determined relative to GAPDH. "P<0.05 vs. 14 days. GFP, green fluorescent protein; AAV9, adeno-associated virus 9; Con, control; ApoE, apolipoprotein E.
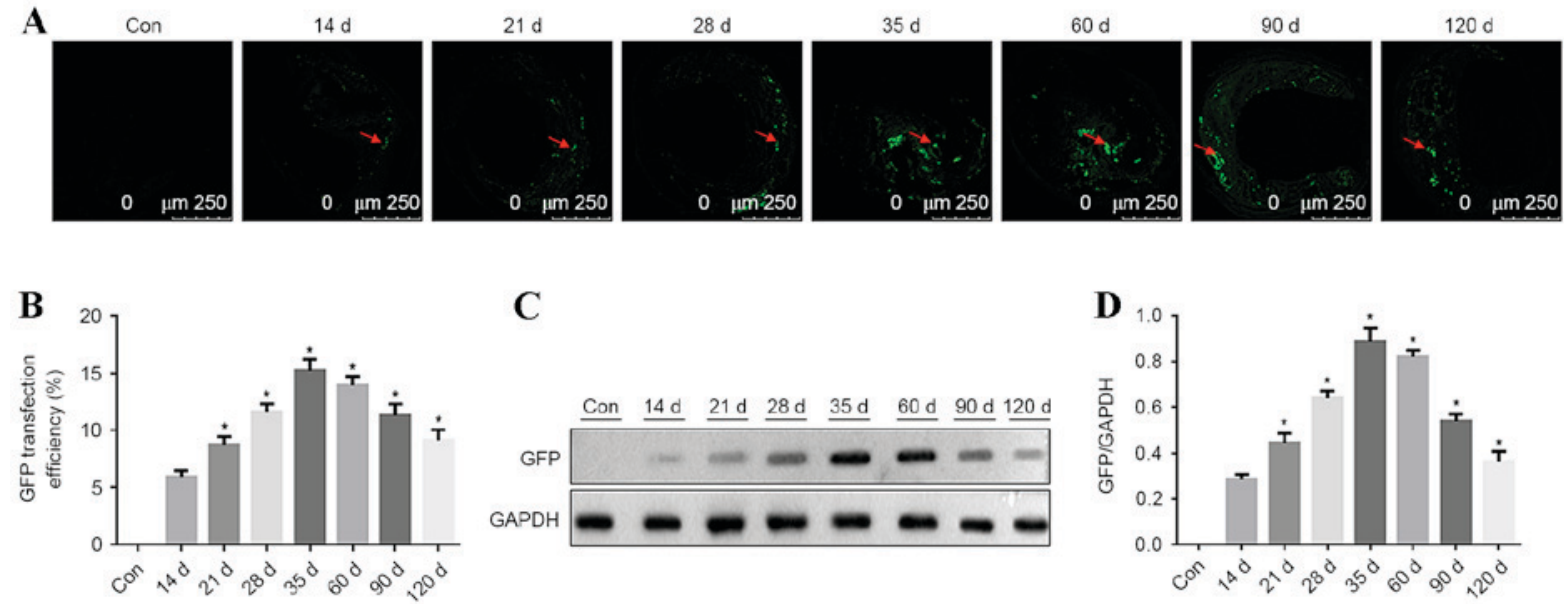

Figure 4. Temporal changes in AAV9-mediated GFP expression in carotid atherosclerotic plaques in $\mathrm{ApoE}^{-/}$mice. (A) Representative confocal microscopy images of AAV9-CMV-mediated GFP expression in the carotid atherosclerotic plaques (Sky Blue was used) at different time points following viral injection. Scale bar, $250 \mu \mathrm{m}$. The red arrows indicate areas of GFP expression, which are green. (B) Quantitative analysis of GFP protein percentage values mean fluorescence intensity (transfection efficiency) in atherosclerotic plaques compared with the whole plaques. ${ }^{*} \mathrm{P}<0.05$ vs. previous time point. (C) GFP expression was quantified by western blotting at different time points following gene delivery ( $\mathrm{n}=5$ per group). (D) Quantitative analysis of GFP protein expression. Expression level of the GFP protein was determined relative to GAPDH. "P<0.05 vs. previous time point. GFP, green fluorescent protein; Con, control; ApoE, apolipoprotein E.

expression levels detected by western blotting were low. This result is consistent with of a previous study as it is challenging to transduce the arterial wall using AAV (29). In a previous study, aorta smooth muscle cells were poorly transduced, changing the route of administration (from intravenous to intra-arterial injections) did not increase the AAV9 transfection efficiency in the aorta of newborn mice (14). Notably, it was determined that rAAV9 transfection of the mouse aorta was more efficient in adult mice, highlighting the importance of designing age-specific gene therapy applications for the aorta. This age-specific difference in the aorta may due to the fact that that adult mice have more vascular endothelial cells that can be infected by rAAV9 compared with newborn mice. Previous studies have stated hypotheses regarding the underlying mechanisms for the rate-limiting barriers in systematic rAAV9-mediated gene transduction (15-18). The present study also determined that rAAV9-CMV-GFP may be transfected into atherosclerotic lesions, where it induced persistent gene expression for up to 120 days. Immunofluorescence staining and western blotting for GFP indicated peak expression at 35 days, indicating a time-dependent process. These findings are in agreement with our previous study, which determined that rAAV9-CMV-GFP may efficiently transfect the heart for a long time, with peak expression occurring at the same time point of 35 days (21). This time delay in peak expression may occur as in order for rAAV9 to mediate transgene expression, single-stranded DNA should be converted into double-stranded DNA $(25,30)$. This process may lead to the delayed expression of genes transfected with rAAV9 compared with other gene transduction vectors, such as adenovirus and lentivirus (7). Notably, transfection with rAAV9-CMV-GFP was more challenging in $\mathrm{C} 57 / 6 \mathrm{~B}$ mice, whereas the atherosclerotic lesions in the $\mathrm{ApoE}^{-/}$mice were efficiently transfected at higher level and persisted for longer. The exact molecular mechanism underlying this process remains to be elucidated. It is possible that the $\mathrm{ApoE}^{-/-}$mouse lesions contained a greater number of cells, such as smooth muscle and endothelial cells, and macrophages compared with the corresponding vasculature in the C57/6B mice. It may also be that the greater organ and cell tropism of the CMV promoter may lead to this difference (20). Future investigations are required to elucidate the mechanism to improve clinical gene therapy. 

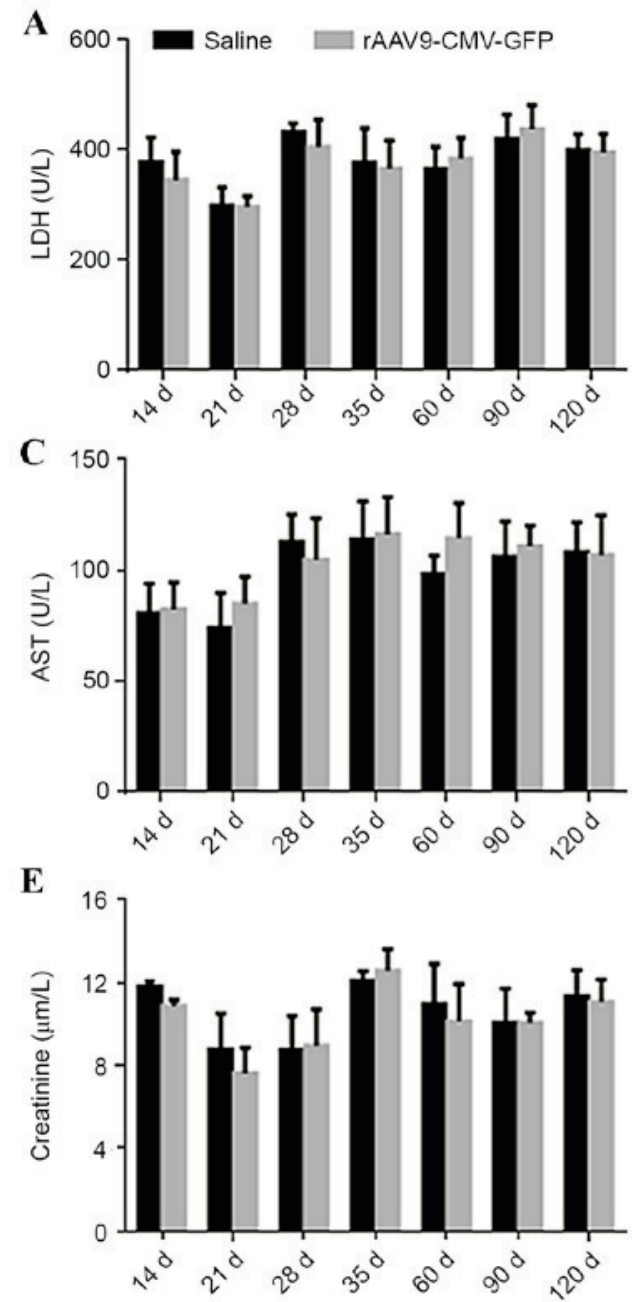
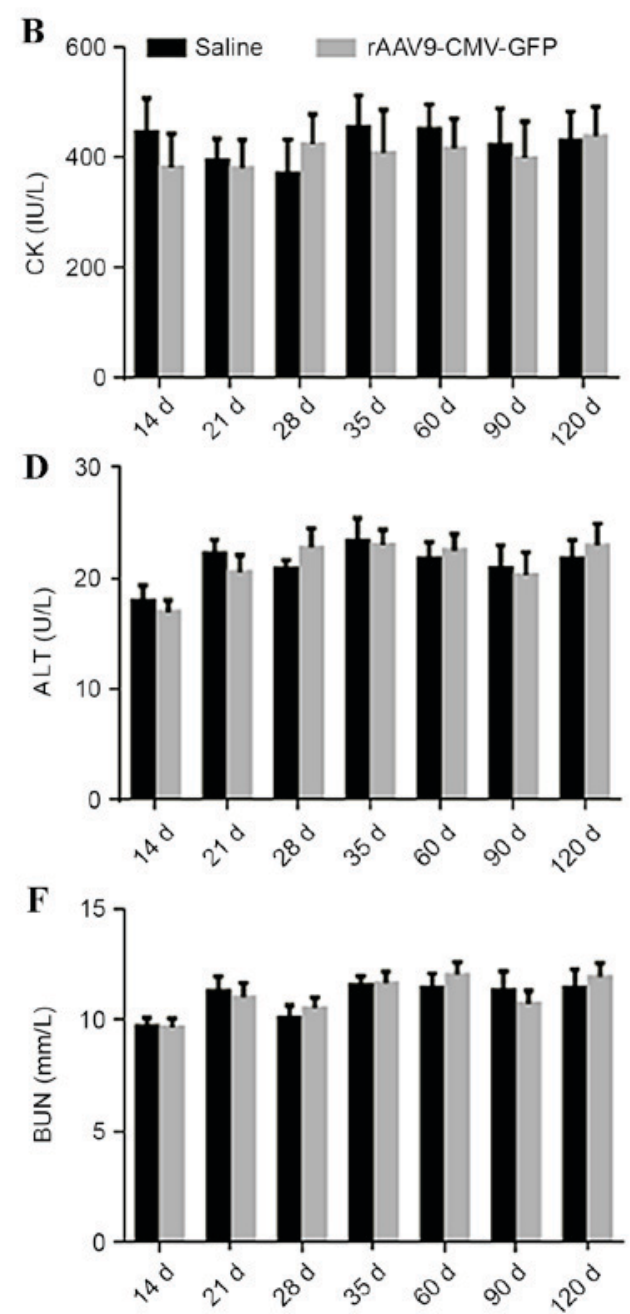

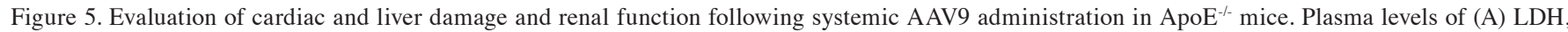
(B) CK, (C) AST, (D) ALT, (E) creatinine and (F) BUN from 14 to 120 days following systemic AAV9-CMV vector administration and normal saline injection (control) ( $\mathrm{n}=5$ per group). LDH, lactate dehydrogenase; CK, creatine kinase; AST, aspartate aminotransferase; ALT, alanine aminotransferase; BUN, blood urea nitrogen; rAAV-CMV-GFP, recombinant adeno-associated virus 9-cytomegalovirus-green fluorescent protein; ApoE, apolipoprotein E.
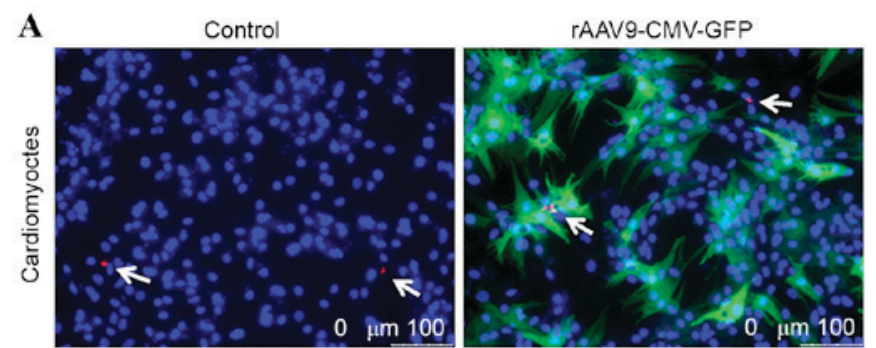

C

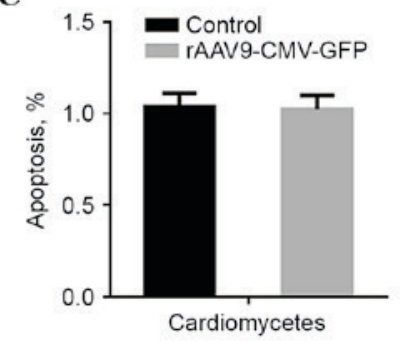

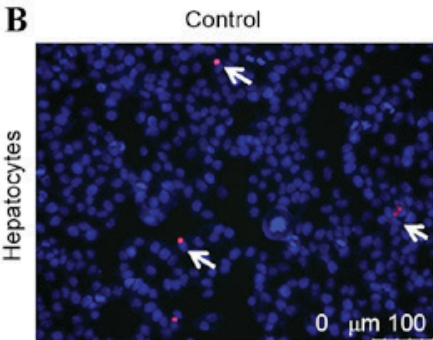
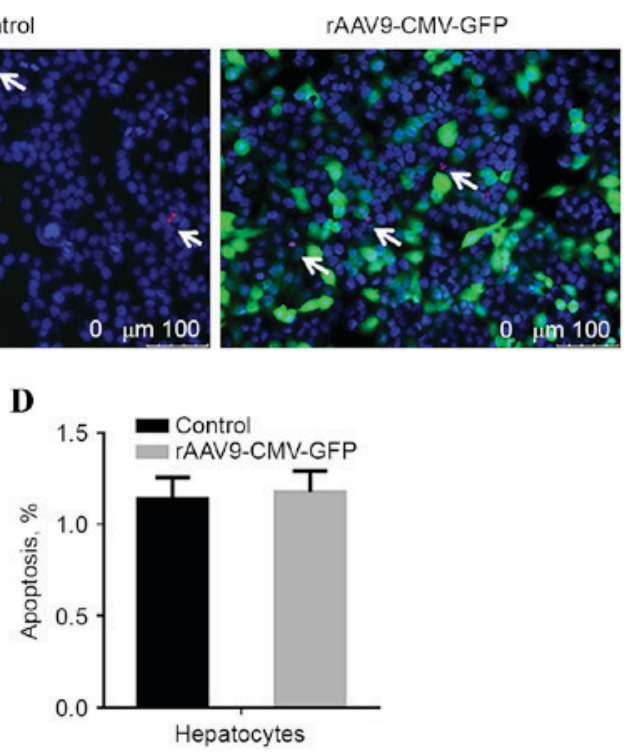

Figure 6. Apoptosis assay of cardiomyocytes and hepatocytes following AAV9-CMV-GFP transduction. AAV9-CMV-GFP transduction of (A) cardiomyocytes and (B) hepatocytes for 4 days did not lead to significant changes in apoptosis. Arrows indicate TUNEL-positive cells, which are red. Scale bar, $100 \mu \mathrm{m}$. Quantitative analysis of TUNEL-positive cells for (C) cardiomyocytes and (D) hepatocytes. rAAV-CMV-GFP, recombinant adeno-associated virus 9-cytomegalovirus-green fluorescent protein; TUNEL, terminal deoxynucleotidyl transferase dUTP nick end labeling. 
The present study also determined that the rAAV9 vector delivery system did not lead to significant cardiac, liver or kidney damage compared with the control group based on the serum levels of LDH, CK, AST, ALT, creatinine and BUN detected. No significant difference was identified in the number of TUNEL-positive cardiomyocytes and hepatic cells between transfected and non-transfected control groups. Thus, a high dose of the rAAV9 vector had no marked cellular cytotoxic effects. These findings indicated that the single-stranded rAAV9-CMV vector may be an optimal and safe choice for gene therapy of chronic heart disease, particularly for advanced atherosclerosis. However, the present study had certain limitations. Initially, no comparisons between different doses of rAAV9-CMV were made to identify the optimal dose, the recommended dose reported in a previous study was used instead. Subsequently, the type of cells transfected by rAAV9-CMV was not identified, further investigations are required to determine the transfected cell types to improve targeted gene therapy.

In conclusion, the present study demonstrated that the rAAV9 viral vector produced using the rBac system may be effectively expressed in atherosclerotic plaques. As atherosclerosis is a chronic condition, long-term gene expression is required. The current study indicated that this may be achieved using an rAAV9-based vector. Systemic administration of rAAV9 or direct transduction of atherosclerotic plaques did not induce major organ injury and apoptosis. rAAV9 vectors with a CMV promoter may be used for efficient gene transfer into atherosclerotic plaques in the future.

\section{Acknowledgements}

The present study was supported by a grant from the National Natural Science Foundation of China (grant no. 81160042).

\section{References}

1. Lewis SJ: Prevention and treatment of atherosclerosis: A practitioner's guide for 2008. Am J Med 122 (1 Suppl): S38-S50, 2009

2. de Nooijer R, Verkleij CJ, von der Thüsen JH, Jukema JW, van der Wall EE, van Berkel TJ, Baker AH and Biessen EA: Lesional overexpression of matrix metalloproteinase- 9 promotes intraplaque hemorrhage in advanced lesions but not at earlier stages of atherogenesis. Arterioscler Thromb Vasc Biol 26: 340-346, 2006

3. Zadelaar AS, von der Thüsen JH, Boesten LS, Hoeben RC, Kockx MM, Versnel MA, van Berkel TJ, Havekes LM, Biessen EA and van Vlijmen BJ: Increased vulnerability of pre-existing atherosclerosis in ApoE-deficient mice following adenovirus-mediated Fas ligand gene transfer. Atherosclerosis 183 244-250, 2005

4. Von Der Thüsen JH, Kuiper J, Fekkes ML, De Vos P, Van Berkel TJ and Biessen EA: Attenuation of atherogenesis by systemic and local adenovirus-mediated gene transfer of interleukin-10 in LDLr-/- mice. FASEB J 15: 2730-2732, 2001.

5. White K, Büning H, Kritz A, Janicki H, McVey J, Perabo L, Murphy G, Odenthal M, Work LM, Hallek M, et al: Engineering adeno-associated virus 2 vectors for targeted gene delivery to atherosclerotic lesions. Gene Ther 15: 443-451, 2008.

6. Skubis-Zegadło J, Stachurska A and Małecki M: Vectrology of adeno-associated viruses (AAV). Med Wieku Rozwoj 17: 202-206, 2013.

7. Pacak CA and Byrne BJ: AAV vectors for cardiac gene transfer: Experimental tools and clinical opportunities. Mol Ther 19: 1582-1590, 2011.

8. White SJ, Nicklin SA, Büning H, Brosnan MJ, Leike K, Papadakis ED, Hallek M and Baker AH: Targeted gene delivery to vascular tissue in vivo by tropism-modified adeno-associated virus vectors. Circulation 109: 513-519, 2004.
9. Work LM, Büning H, Hunt E, Nicklin SA, Denby L, Britton N, Leike K, Odenthal M, Drebber U, Hallek M and Baker AH: Vascular bed-targeted in vivo gene delivery using tropism-modified adeno-associated viruses. Mol Ther 13: 683-693, 2006.

10. Nathwani AC, Davidoff A, Hanawa H, Zhou JF, Vanin EF and Nienhuis AW: Factors influencing in vivo transduction by recombinant adeno-associated viral vectors expressing the human factor IX cDNA. Blood 97: 1258-1265, 2001.

11. Koeberl DD, Alexander IE, Halbert CL, Russell DW and Miller AD: Persistent expression of human clotting factor IX from mouse liver after intravenous injection of adeno-associated virus vectors. Proc Natl Acad Sci USA 94: 1426-1431, 1997.

12. Aikawa R, Huggins GS and Snyder RO: Cardiomyocyte-specific gene expression following recombinant adeno-associated viral vector transduction. J Biol Chem 277: 18979-18985, 2002.

13. Prasad KM, Xu Y, Yang Z, Toufektsian MC, Berr SS and French BA: Topoisomerase inhibition accelerates gene expression after adeno-associated virus-mediated gene transfer to the mammalian heart. Mol Ther 15: 764-771, 2007.

14. Bostick B, Ghosh A, Yue Y, Long C and Duan D: Systemic AAV-9 transduction in mice is influenced by animal age but not by the route of administration. Gene Ther 14: 1605-1609, 2007.

15. Gregorevic P, Blankinship MJ, Allen JM, Crawford RW, Meuse L, Miller DG, Russell DW and Chamberlain JS: Systemic delivery of genes to striated muscles using adeno-associated viral vectors. Nat Med 10: 828-834, 2004.

16. Inagaki K, Fuess S, Storm TA, Gibson GA, Mctiernan CF, Kay MA and Nakai H: Robust systemic transduction with AAV9 vectors in mice: Efficient global cardiac gene transfer superior to that of AAV8. Mol Ther 14: 45-53, 2006.

17. Pacak CA, Mah CS, Thattaliyath BD, Conlon TJ, Lewis MA, Cloutier DE, Zolotukhin I, Tarantal AF and Byrne BJ: Recombinant adeno-associated virus serotype 9 leads to preferential cardiac transduction in vivo. Circ Res 99: e3-e9, 2006.

18. Wang Z, Zhu T, Qiao C, Zhou L, Wang B, Zhang J, Chen C, Li J and Xiao X: Adeno-associated virus serotype 8 efficiently delivers genes to muscle and heart. Nat Biotechnol 23: 321-328, 2005.

19. Ghosh A, Yue Y, Long C, Bostick B and Duan D: Efficient whole-body transduction with trans-splicing adeno-associated viral vectors. Mol Ther 15: 750-755, 2007.

20. Pacak CA, Sakai Y, Thattaliyath BD, Mah CS and Byrne BJ: Tissue specific promoters improve specificity of AAV9 mediated transgene expression following intra-vascular gene delivery in neonatal mice. Genet Vaccines Ther 6: 13, 2008.

21. Chen BD, He CH, Chen XC, Pan S, Liu F, Ma X, Li XM, Gai MT Tao J, Ma YT, et al: Targeting transgene to the heart and liver with AAV9 by different promoters. Clin Exp Pharmacol Physiol 42: 1108-1117, 2015.

22. Virag T, Cecchini S and Kotin RM: Producing recombinant adeno-associated virus in foster cells: Overcoming production limitations using a baculovirus-insect cell expression strategy. Hum Gene Ther 20: 807-817, 2009.

23. Chen H: Intron splicing-mediated expression of AAV Rep and Cap genes and production of AAV vectors in insect cells. Mol Ther 16: 924-930, 2008.

24. Chen H: Exploiting the intron-splicing mechanism of insect cells to produce viral vectors harboring toxic genes for suicide gene therapy. Mol Ther Nucleic Acids 1: e57, 2012.

25. Geisler A, Jungmann A, Kurreck J, Poller W, Katus HA, Vetter R, Fechner H and Müller OJ: microRNA122-regulated transgene expression increases specificity of cardiac gene transfer upon intravenous delivery of AAV9 vectors. Gene Ther 18: 199-209, 2011.

26. Livak KJ and Schmittgen TD: Analysis of relative gene expression data using real-time quantitative PCR and the 2(-Delta Delta C(T)) Method. Methods 25: 402-408, 2001.

27. Guo W, Wong S and Bhasin S: AAV-mediated administration of myostatin pro-peptide mutant in adult Ldlr null mice reduces diet-induced hepatosteatosis and arteriosclerosis. PLoS One 8: e71017, 2013.

28. Foster K, Graham IR, Otto A, Foster H, Trollet C, Yaworsky PJ, Walsh FS, Bickham D, Curtin NA, Kawar SL, et al: Adeno-associated virus-8-mediated intravenous transfer of myostatin propeptide leads to systemic functional improvements of slow but not fast muscle. Rejuvenation Res 12: 85-94, 2009.

29. Baker AH, Kritz A, Work LM and Nicklin SA: Cell-selective viral gene delivery vectors for the vasculature. Exp Physiol 90: 27-31, 2005.

30. Wang Z, Ma HI, Li J, Sun L, Zhang J and Xiao X: Rapid and highly efficient transduction by double-stranded adeno-associated virus vectors in vitro and in vivo. Gene Ther 10: 2105-2111, 2003. 